\title{
MHD simulations of cool core clusters
}

\author{
Frederico Stasyszyn and Klaus Dolag \\ MPI for Astrophysics, Karl-Schwarzchild-Str. 1, D-85741 Garching, Germany \\ e-mail: fstasys@mpa-garching.mpg.de
}

\begin{abstract}
Using Smoothed Particle Magneto Hydrodynamics (Dolag \& Stasyszyn), we study the effects of magnetic fields in galaxy clusters with the aim to infer their dynamical role within the cool core region. Therefore we we investigate the role of regularization as well as divergence cleaning schemes (Stasyszyn \& Dolag 2009). We run cosmological simulations of a reference cluster in order to evaluate our various implementation in a realistic scenario. The preliminary results indicate that the final magnetic field profile in the simulations depends only on the amount of artificial dissipation, but not in the amount of numerical $\operatorname{div}(B)$ present in the different implementation. We also present first results from simulations which are including radiative cooling and star formation. Even at the this low resolution we find a strong additional amplification of the magnetic field within the cool core region indicating that magnetic pressure could become comparable to the thermal pressure in theses regimes.
\end{abstract}

Keywords. MHD - methods: n-body simulations - galaxies: clusters - galaxies: magnetic fields

\section{Introduction}

The SPH MHD implementation (Dolag \& Stasyszyn) in Gadget (Springel) allows to use several regularization schemes to stabilize the numerical MHD implementation and to suppress the grow of irregularities as well as numerical $\operatorname{div}(B)$ in the simulations. We extended these regularization schemes by a $\operatorname{div}(B)$ cleaning method originally proposed by Dedner. In this method a scalar field is evolved, which propagates and damps the numerical divergence. To obtain good stability of the Dedner method, some modifications have been made. The most important are the suppression of the correction within shocks fronts and a self consistent formulation which includes the corresponding terms in the MHD equations. We performed several tests as the Fast Rotor (see Price \& Monaghan, Lodrillo \& Del Zanna) and Orzang-Tang Vortex (see Price \& Monaghan, Orzang \& Tang, Balsara) to demonstrate the performance and robustness of our implementation and all MHD SPH simulations compare well with the results obtained with Athena (Stone). In our tests, the regularization schemes which involve some numerical dissipation are very effective in suppressing irregularities evolving in the magnetic field but also
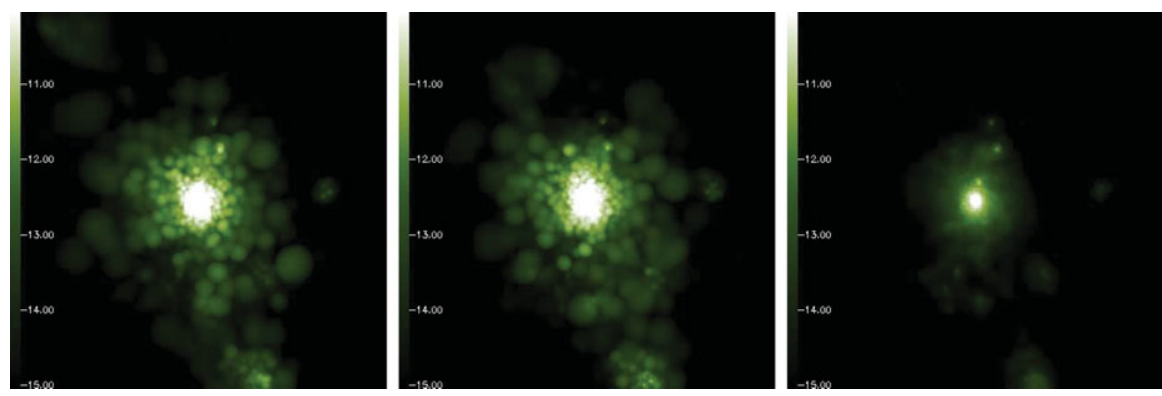

Figure 1. $B^{2}$ of a slice through our test cluster for non radiative simulations. The normal implementation at left, Dedner in the center and Euler potentials in the right. 

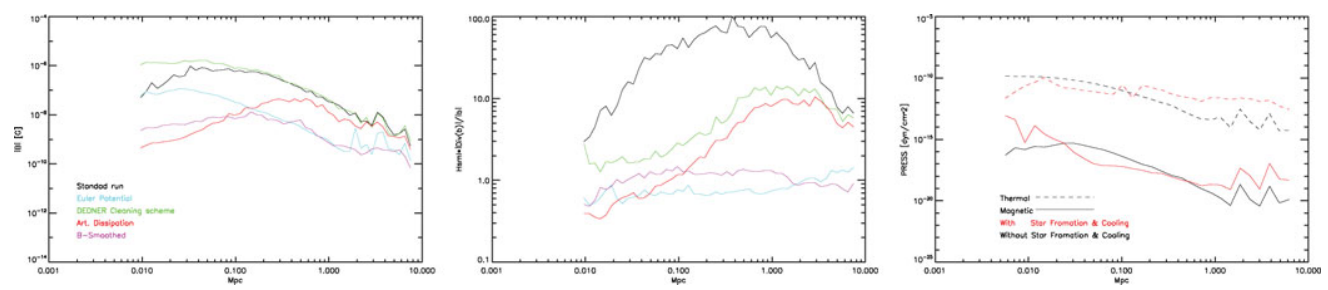

Figure 2. The profiles for the $|B|$ (left), $h *|\operatorname{div}(B)| /|B|$ (center), thermal and magnetic pressure for the Euler Potential runs with and without cooling (right).

tend to significantly smear sharp features. In contrary the new $\operatorname{div}(B)$ cleaning is able to significantly suppress the presence of numerical $\operatorname{div}(B)$ without any visible smearing of sharp features. For some test we also used so called Euler Potential (see Rosswog \& Price), where $B$ is calculated from the evolution of two potentials.

\section{The reference cluster}

In the figure 1 we show a slice of $\left|B^{2}\right|$ from or reference cluster, simulated with the different schemes. The mass of the cluster is $\approx 10^{14} M_{\odot} / h$ and the particles masses are $1.1 \times 10^{9} M_{\odot} / h$ and $1.7 \times 10^{8} M_{\odot} / h$ for the dark matter and the gas particles respectively. In Fig. 2 we show the profiles of the magnetic field, a measure of $\operatorname{div}(B)$ and the pressure profiles of several runs for this cluster. The magnetic field profiles show how the regularization schemes which involve different numerical dissipation are (over-)suppressing the magnetic field in different regions. In contrary, the Dedner scheme results in a quite similar profile to the run without regularization, but with significant less numerical $\operatorname{div}(B)$. First test runs using the Euler Potentials with and without cooling and star formation indicate that magnetic pressure could become comparable to the thermal one in the cluster center.

\section{Conclusions}

We implemented a SPH-MHD method with different regularization and divergence cleaning schemes in the cosmological code Gadget3. We explore the these methods in a cosmological frame using a low mass cluster. We demonstrate that the resulting magnetic field profiles are sensitive to the amount of numerical dissipation involved in the different regularization schemes. Using a $\operatorname{div}(B)$ cleaning scheme further demonstrate that the resulting magnetic field profile is not driven by $\operatorname{div}(B)$ errors. First test simulations including cooling and star-formation indicate an additional growth of magnetic field in cool core regions, where the magnetic pressure can eventually become significant.

\section{References}

Balsara, D. S. et al. 1998, JCP 149, 270

Borbe, S. et al. 2001, ApJ 561, 82

Dedner, J. et al. 2002, JCP 175, 645

Dolag, K. \& Stasyszyn, F. 2008, arXiv arXiv:0807.3553

Lodrillo, P. \& Del Zanna, L. 2000, ApJ 530, 508

Orzang, S. \& Tang, C. 1978, JFM 90, 128

Price, D. \& Monaghan, J. 2005, MNRAS 264, 384

Rosswog, S. \& Price, D. 2007, MNRAS 379, 915

Springel, V. 2005, MNRAS 364, 1105

Stasyszyn, F. \& Dolag K., 2009, MNRAS, in prep.

Stone, J. et al. 2008, ApJS 178, 137 